\title{
Dark-bright exciton spin-flip rates of quantum dots determined by a modified local density of optical states
}

\author{
Lodahl, Peter; Johansen, Jeppe; Julsgaard, Brian; Hvam, Jørn Märcher
}

Published in:

Conference abstract series, CLEO/Europe - EQEC

Link to article, DOI:

10.1109/CLEOE-EQEC.2009.5192256

Publication date:

2009

Document Version

Publisher's PDF, also known as Version of record

Link back to DTU Orbit

Citation (APA):

Lodahl, P., Johansen, J., Julsgaard, B., \& Hvam, J. M. (2009). Dark-bright exciton spin-flip rates of quantum dots determined by a modified local density of optical states. In Conference abstract series, CLEO/Europe - EQEC IEEE. https://doi.org/10.1109/CLEOE-EQEC.2009.5192256

\section{General rights}

Copyright and moral rights for the publications made accessible in the public portal are retained by the authors and/or other copyright owners and it is a condition of accessing publications that users recognise and abide by the legal requirements associated with these rights.

- Users may download and print one copy of any publication from the public portal for the purpose of private study or research.

- You may not further distribute the material or use it for any profit-making activity or commercial gain

- You may freely distribute the URL identifying the publication in the public portal 


\title{
Dark-bright exciton spin-flip rates of quantum dots determined by a modified local density of optical states
}

\author{
Peter Lodahl, Jeppe Johansen, Brian Julsgaard, and Jorn M. Hvam \\ DTU Fotonik, Department of Photonics Engineering, \\ Technical University of Denmark, Ørsteds Plads 343, DK-2800 Kgs. Lyngby, \\ Denmark
}

Semiconductor quantum dots (QDs) are popular light sources in contemporary solid-state quantum optics and used extensively for, e.g., all-solid-state single-photon sources [1,2]. In order to design and understand such devices, a detailed understanding of the optical properties of QDs is needed. QDs are not ideal two-level emitters, and effects of non-radiative processes, dephasing, and exciton fine structure must be carefully considered. Here we investigate the influence of dark excitons on the radiative dynamics of QDs. Dark excitons have total angular momentum of 2 and contribute to the fine structure of the exciton ground state. As opposed to bright excitons that have total angular momentum 1, dark excitons cannot recombine directly via electric dipole transitions. However, slow recombination does take place since the dark exciton can undergo a spin-flip process thereby transferring it into a bright exciton, see Fig. 1(a).

We demonstrate a technique to extract the dark-bright exciton spin-flip rate based on time-resolved detection of spontaneous emission. Using the modified local density of optical states of a GaAs-air interface and its known dependence on distance (z), it was recently shown that the bright exciton radiative and non-radiative processes could be fully unravelled [3]. Armed with this knowledge, the spin-flip rate can be extracted. The spontaneous emission decay curves are found to be bi-exponential and the dark excitons contribute to the slow component with a rate dominated by non-radiative processes. The spin-flip rate can be extracted reliably from the ratio of the amplitudes of the slow and fast components.

Fig. 1(b) shows the spin-flip rate as a function of distance to the interface for six different emission energies. A typical spin-flip time is found to be around $100 \mathrm{~ns}$, but pronounced dependences on emission energy and distance to the interface are observed. This gives important information as to the nature of the spin-flip process. It has been proposed that dark-bright spin flips can be induced by the interplay of exciton exchange interaction and phonons [4]. We discuss our results in view of this model, which suggests that the increase close to the interface is due to enhanced phonon interaction. Furthermore, the sensitive dependence on QD emission energy is interpreted as due to the dependence of the exciton exchange interaction on the QD size.

(a)

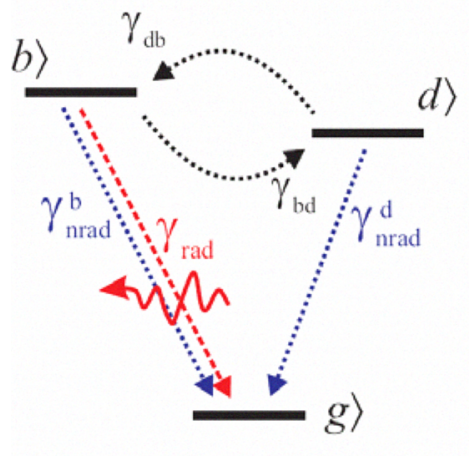

(b)

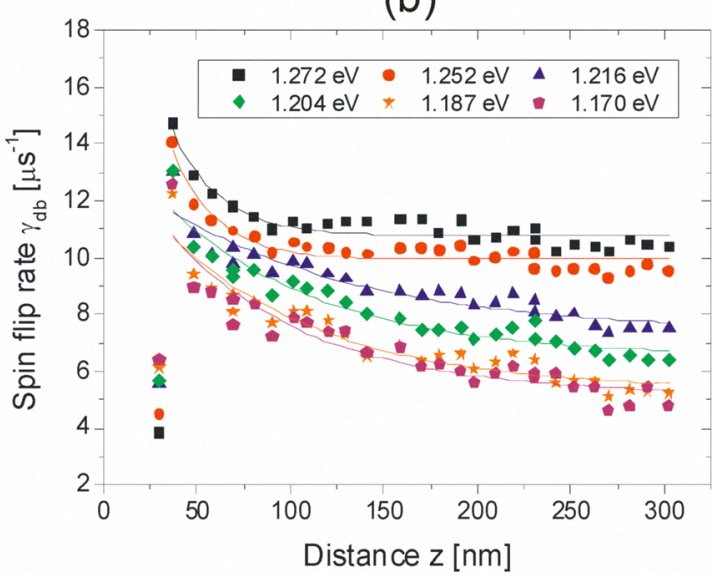

Fig. 1 (a) The QD level scheme where $b$ and $d$ are the bright and dark exciton states, respectively, while $g$ is the state corresponding to no exciton in the QD. The bright state can recombine through radiative and non-radiative processes while the dark state can either decay by non-radiative recombination or undergo a spin-flip transition (rate $\gamma_{\mathrm{db}}$ ) to a bright exciton. (b) Measured spin-flip rate as a function of distance (z) to the GaAs-air interface at six different QD emission energies. The spin-flip rate is modelled with an exponential decay in $\mathrm{z}$ (solid curves).

\section{References}

[1] B. Lounis and M. Orrit, "Single-photon sources", Rep. Prog. Phys. 68, 1129 (2005).

[2] T. Lund-Hansen, S. Stobbe, B. Julsgaard, H. Thyrrestrup, T. Sünner, M. Kamp, A. Forchel, and P. Lodahl, "Experimental realization of highly efficient broadband coupling of single quantum dots to a photonic crystal waveguide", Phys. Rev. Lett. 101, 113903 (2008).

[3] J. Johansen, S. Stobbe, I.S. Nikolaev, T. Lund-Hansen, P.T. Kristensen, J.M. Hvam, W.L. Vos, and P. Lodahl, "Size dependence of the wavefunction of self-assembled InAs quantum dots from time-resolved optical measurements", Phys. Rev. B 77, 073303 (2008).

[4] K. Roszak, V.M. Axt, T. Kuhn, and P. Machnikowski, "Exciton spin decay in quantum dots to bright and dark states", Phys. Rev. B 76, $195324(2007)$ 